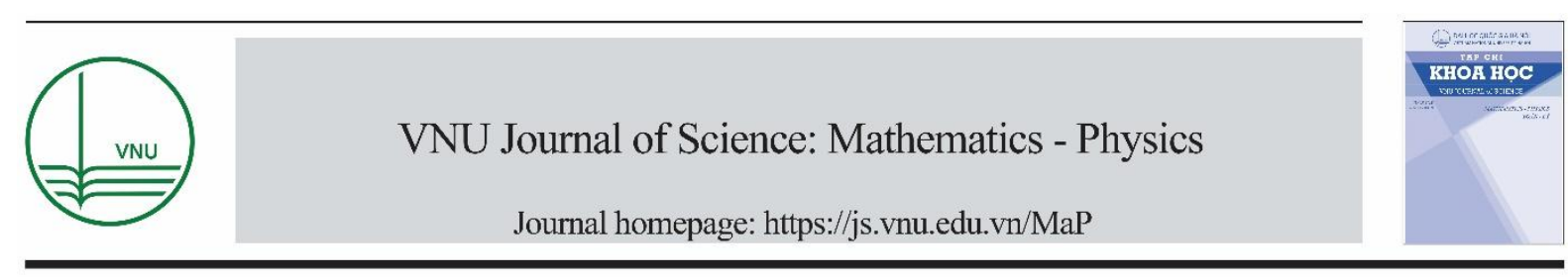

Original Article

\title{
Gas Sensors Based on U-shaped Graphene Nanoribbons: A first-principles Study
}

\author{
Nguyen Tien Cuong* \\ Faculty of Physics, VNU University of Science, Hanoi, Vietnam
}

Received 11 January 2020

Revised 19 February 2020; Accepted 26 February 2020

\begin{abstract}
In this paper, I present first-principle calculations which are performed by using combination of DFT and NEGF methods to investigate the adsorption of $\mathrm{CO}_{2}$ and $\mathrm{NH}_{3}$ molecules on straight and U-shaped GNRs junctions. The density of state, molecular orbital, adsorption energy, transmission spectrum, and current-voltage characteristics were analyzed. It is found that the adsorption of $\mathrm{CO}_{2}$ and $\mathrm{NH}_{3}$ on straight GNRs at the dangling bond edges exhibit chemical interaction behavior with high adsorption energy. Besides, for U-shaped ZGRNs without adsorption, the states around Fermi level are quasi-bound states localized at the zigzag edges. The electrons at these localized states are almost confined and do not contribute on conductance. However, for U-shaped GRNs with $\mathrm{CO}_{2}$ and $\mathrm{NH}_{3}$ adsorbed on DB armchair edge defects, these states become delocalized on whole of the U-shaped junction. The current-voltage characteristics show that the sensitivity and selectivity of the U-shaped GNRs gas sensors are significantly improved in comparison with the pristine GNR ones.
\end{abstract}

Keywords: DFT, NEGF, U-shaped GRNs, $\mathrm{CO}_{2}$ Adsorbed on Graphene, $\mathrm{NH}_{3}$ Adsorbed on Graphene, Graphene gas sensors.

\section{Introduction}

It is well known that Graphene is attracting massive worldwide interest for nano-scale device applications since its successful fabrication in 2004 [1]. Graphene offer great potential applications for gas sensors due to their high mobility and high surface area to volume ratio [2]. Sensing toxic gas molecules is very important in environmental pollution monitoring and medical applications [3]. The adsorptions of gas molecules on the infinite Graphene surface have been studied theoretically [4]. The

\footnotetext{
*Corresponding author.

Email address: ntcuong@ hus.edu.vn
}

https//doi.org/ 10.25073/2588-1124/vnumap.4454 
adsorption of ammonia $\left(\mathrm{NH}_{3}\right)$ and carbon dioxide $\left(\mathrm{CO}_{2}\right)$ on Graphene sheet have been investigated in experiments $[5,6]$.

The adsorptions of $\mathrm{NH}_{3}$ and $\mathrm{CO}_{2}$ on pristine Graphene Nanoribbons (GNRs) have been reported in our previous paper [7]. This study indicated that both $\mathrm{NH}_{3}$ and $\mathrm{CO}_{2}$ are physically adsorbed on pristine GNRs with low adsorption energies. In other words, the interactions between $\mathrm{CO}_{2}$ and $\mathrm{NH}_{3}$ adsorbed molecules and GRNs are very weak. The results suggest that the sensitivity and selectivity of Graphene-based gas sensors could be improved by introducing the dopant, defect, or modification of electronic structure of Graphene. It is well-known that dangling bond (DB) defects around the vacancy sites or at the tips is very important of gas molecular sensors based on CNTs [8,9]. DB defects would exist at two edges of GNRs. Because two edges of a GNR is equivalent in nature. In this paper, the electronic and transport properties of $\mathrm{NH}_{3}$ and $\mathrm{CO}_{2}$ molecules adsorbed on straight GNRs with double DB defects on two edges are examined first for understanding the nature of such the adsorption.

In another our paper, the electronic properties and quantum transport in U-shaped GNRs have been presented [10]. It is found that the GNRs junctions tend to open a band gap when U-shaped structures were formed due to the formation of quasi-bound states localized at zigzag edges. The obtained results have provided valuable information for designing potential nano-scale devices based on Graphene. Thus, gas sensors based on U-shaped GNRs are designed. The first-principle calculations were performed for investigating the adsorption of $\mathrm{CO}_{2}$ and $\mathrm{NH}_{3}$ molecules on the Ushaped GNRs gas sensors in this paper. The current-voltage curve of a U-shaped ZGNR sensor was calculated for estimating the sensitivity and selectivity of such the U-shaped GNRs gas sensors.

\section{Model and computational methods}

The electronic and transport properties are calculated using the combination of the density functional theory (DFT) and the non-equilibrium Green's function (NEGF) methods. In particular, the electronic properties such as density of state (DOS), molecular orbitals (MOs), adsorption energy were calculated based on the DFT using both Materials Studio/Dmol ${ }^{3}$ and OpenMX packages $[11,12]$. The transport properties such as transmission spectrum, current-voltage characteristics were calculated by using NEGF method which is implemented in the OpenMX package [12].

For $\mathrm{Dmol}^{3}$ calculations, the MOs of the periodical U-shaped GNRs junctions are calculated at the gamma point using the local density approximation (LDA) for the exchange-correlation functional. All-electron calculations were performed with a finite basis set cutoff of $3.5 \AA$, and self-consistent field (SCF) tolerance of $10^{-5} \mathrm{Ha}$.

For OpenMX calculations, a system consisting of a central region connected to left and right leads with infinite size, as shown in Fig. 1(a), is treated by the NEGF method. By considering the two dimensional periodicity in the bc-plane, the system can be cast into a one-dimensional problem. The electronic transport is assumed to occur along the a-axis. All calculations were carried out with in the LDA exchange-correlation functional. The SCF energy convergence criterion is set to $10^{-5} \mathrm{eV}$. In the treatment of pseudo-potentials, the pseudo atomic orbital basis sets of $\mathrm{C} 4.5-\mathrm{s}^{2} \mathrm{p}^{1}$ for carbon and H4.5$\mathrm{s}^{2}$ for hydrogen were used, where in the abbreviation of basis functions such as $\mathrm{C} 4.5-\mathrm{s}^{2} \mathrm{p}^{1}, \mathrm{C}$ stands for the atomic symbol, 4.5 the cutoff radius (Bohr) in the generation by the confinement scheme, and $s^{2} p^{1}$ means the employment of two primitive orbitals for $s$ and one primitive orbital for $p$ [12].

The structural model as shown in Fig. 1(b) was used for calculating the adsorption of $\mathrm{CO}_{2}$ and $\mathrm{NH}_{3}$ on straight GNRs at the dangling bond (DB) edges. The structural model as shown in Fig. 1(c) was used for calculating the electronic and transport properties of U-shaped GNRs junctions without and with adsorption of $\mathrm{CO}_{2}$ and $\mathrm{NH}_{3}$ on the $\mathrm{DB}$ armchair. Where, $h$ is the height of the vertical edges of 
the U-shaped junctions. The distance between vertical edges, $d$, is chosen large enough in order to ignore the horizontal interaction among atoms located at vertical edges. In both cases, the lattice parameters, $c$, along the $c$-axis are chosen large enough to avoid the vertical interactions among atoms located at horizontal edges. For surface models, a vacuum region of $15 \AA$ is embedded along the b-axis to minimize interactions between Graphene surfaces.
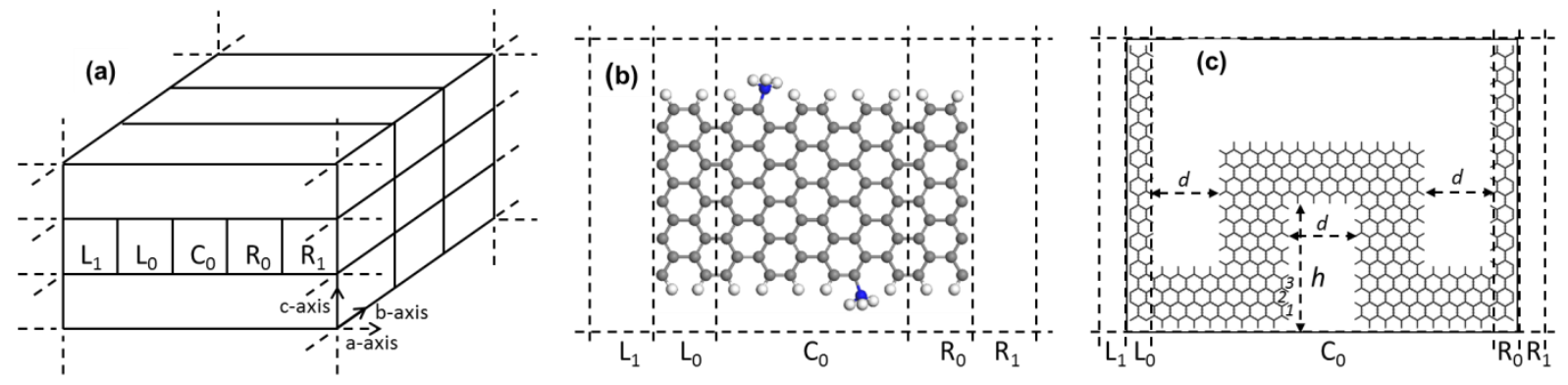

Figure 1. (a) Configuration of the system treated by NEGF method. (b) Structure model of straight GNRs with $\mathrm{NH}_{3}$ adsorption. (c) Structure model of U-shaped GNRs junction

\section{Results and discussion}

\subsection{Adsorption of $\mathrm{CO}_{2}$ and $\mathrm{NH}_{3}$ on GNRs with $\mathrm{DB}$ defects}

The initial structures are built by putting a relax $\mathrm{CO}_{2}\left(\mathrm{NH}_{3}\right)$ molecule on GNRs at the dangling bond edges with bonding length of $1.50 \AA$. The optimized structures are shown in the Fig. 2. We can see that chemical bonds are formed in the adsorbed structures. In comparison with before adsorption, the hexagonal rings, which were adsorbed $\mathrm{CO}_{2}\left(\mathrm{NH}_{3}\right)$ molecules, are slightly distorted. The results of $\mathrm{CO}_{2}$ molecule adsorbed on ZGNR, Fig. 2(a), show that the $\mathrm{C}$ atom is closer to the ribbon surface than the $\mathrm{O}$ atoms and the $\mathrm{C}-\mathrm{C}$ bond length is $1.51 \AA$. The bond length and the angle of the adsorbed $\mathrm{O}-\mathrm{C}-\mathrm{O}$ molecule are $1.245 \AA$ and $129.8^{\circ}$, respectively. $\mathrm{NH}_{3}$ molecule attach to the ZGNR via the $\mathrm{N}$ atom as shown in Fig. 2(b). The length of $\mathrm{N}-\mathrm{C}$ bond is $1.18 \AA$. For AGNRs cases, the geometry of $\mathrm{CO}_{2}$ adsorbed on the ribbon edge is shown in the Fig. 2(c). The C-C and C-O distances are $1.492 \AA$ and $1.094 \AA$, respectively, and the $\mathrm{C}-\mathrm{O}-\mathrm{C}$ angle is $128.59^{\circ}$. The adsorbed $\mathrm{NH}_{3}$ molecule sits $1.492 \AA$ away from the edge carbon atom. The H-N distance is $0.94 \AA$.
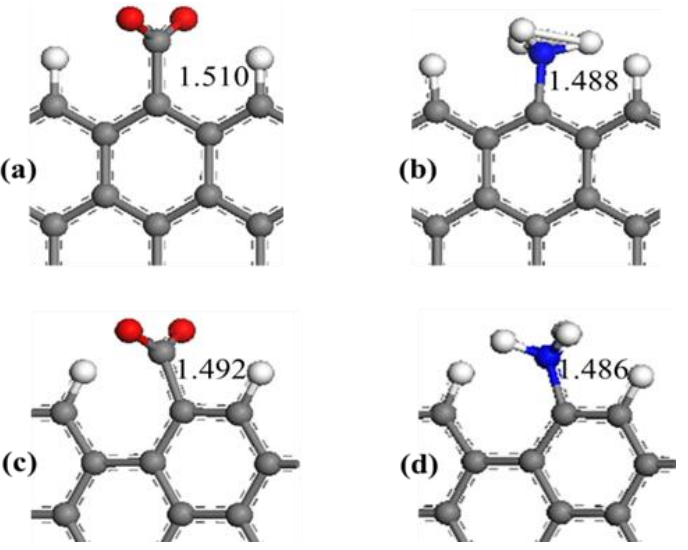

Figure 2. Optimized strudutres of (a) $\mathrm{CO}_{2}$, (b) $\mathrm{NH}_{3}$ adsorbed at DB edges of ZGNRs;

(c) $\mathrm{CO}_{2}$, (d) $\mathrm{NH}_{3}$ adsorbed at DB edges of AGNRs. 
The adsorption energy is defined as formula (1) and shows in the Table 1. Where $E_{\text {GRN }+ \text { Gas }}$ is the total energy of the GNR with gas molecule adsorption. $E_{G R N}$ and $E_{G a s}$ are the energies of isolated GNR and isolated corresponding gas molecule, respectively. In general, a negative $\Delta E_{\text {Ads }}$ indicates that the molecule adsorption is exothermic and thus the adsorption system is energetically stable.

$$
\Delta E_{A d s}=E_{G R N+G a s}-\left(E_{G R N}+E_{G a s}\right)
$$

Table 1. Adsorption energies (eV) of $\mathrm{CO}_{2}$ and $\mathrm{NH}_{3}$ on GRNs with DB

\begin{tabular}{lll}
\hline & $\mathrm{CO}_{2}$ & $\mathrm{NH}_{3}$ \\
\hline AGNR & -3.574 & -1.388 \\
ZGRN & -2.917 & -1.475 \\
\hline
\end{tabular}

Unlike the adsorption of gas molecules on the surface of pristine GNRs which is presented in our previous study [7], the adsorption energies in these cases are quite large. It means that the adsorptions are strong chemisorptions.

Fig. 3 shows the density of states and corresponding transmission spectra of AGNR with $\mathrm{CO}_{2}$ and $\mathrm{NH}_{3}$ adsorptions at DB edges in comparison with pristine AGNR.
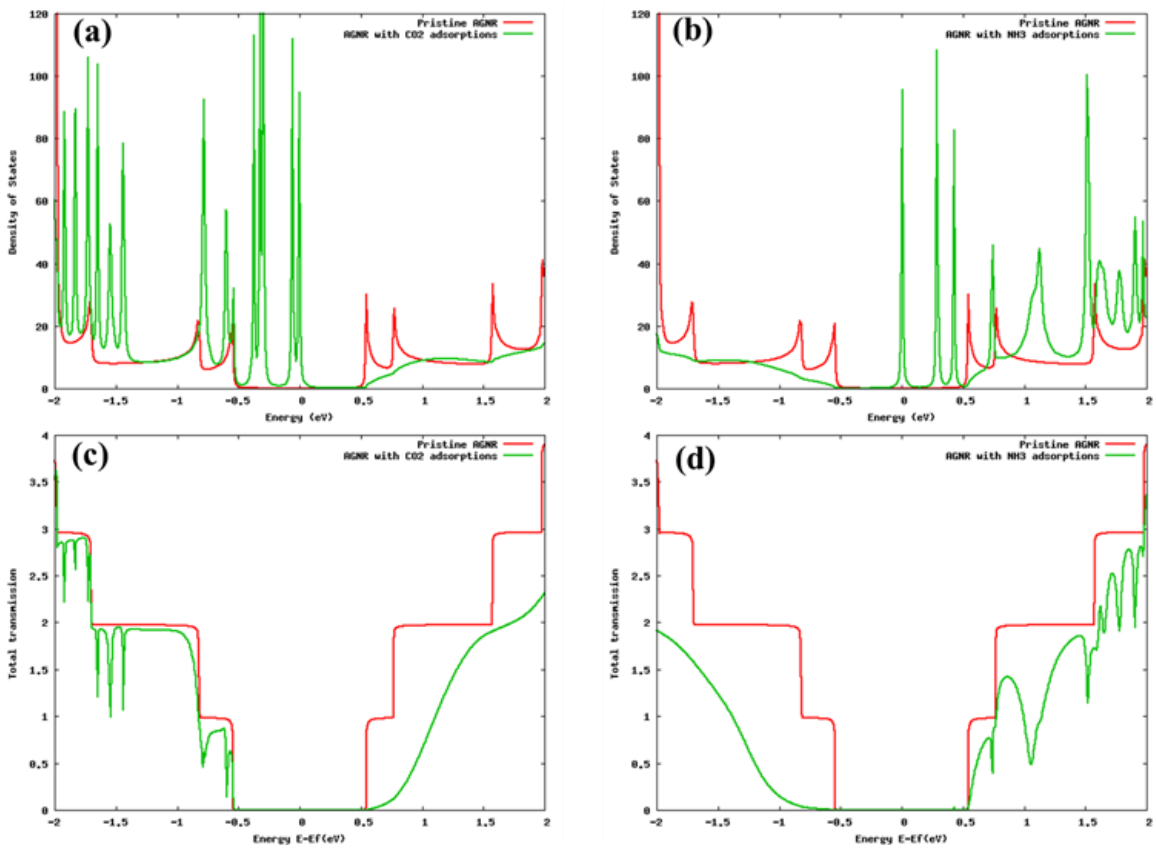

Figure 3. (a), (b) Density of state and (c), (d) corresponding transmission spectra of AGNRs with $\mathrm{CO}_{2}$, and $\mathrm{NH}_{3}$ adsorptions at DB edges, respectively, in comparison with pristine AGNRs

For the pristine AGNR (the red lines), we can see that DOS shows multiple sharp peaks which are caused by lateral quantization of electronic states in the one-dimensional AGNRs as shown in Fig. 3(a) and Fig. 3(b). Therefore, the corresponding transmission spectrum has a step-shape as shown in Fig 3(c) and Fig. 3(d).

For AGNRs adsorbed molecular gases (the green lines), from the DOS, as shown in the Fig. 3(a) and Fig. 3(b), we can see that $\mathrm{CO}_{2}$ adsorbed molecule contributes additional states in the valance band. In opposite, the $\mathrm{NH}_{3}$ molecule contributes additional states in the conductance band. In other words, 
the adsorption of $\mathrm{CO}_{2}$ transfers the system to the p-type semiconductor while the adsorption of $\mathrm{NH}_{3}$ leads to n-type behavior. These results are in good agreement with previous researches $[3,4]$.

\subsection{Electronic and transport properties of $U$-shaped $Z G R N$}

In order to analyze the electronic properties of U-shaped ZGRNs system, we recall our calculations of MOs for the periodical U-shaped GNRs [10]. The highest occupied molecular orbital (HOMO) states and lowest unoccupied molecular orbital (LUMO) states are shown in the Fig. 4(a) and 4(b), respectively. We can see that both of HOMO and LUMO states of the U-shaped ZGRNs are localized at the zigzag edges as quasi-bound states.
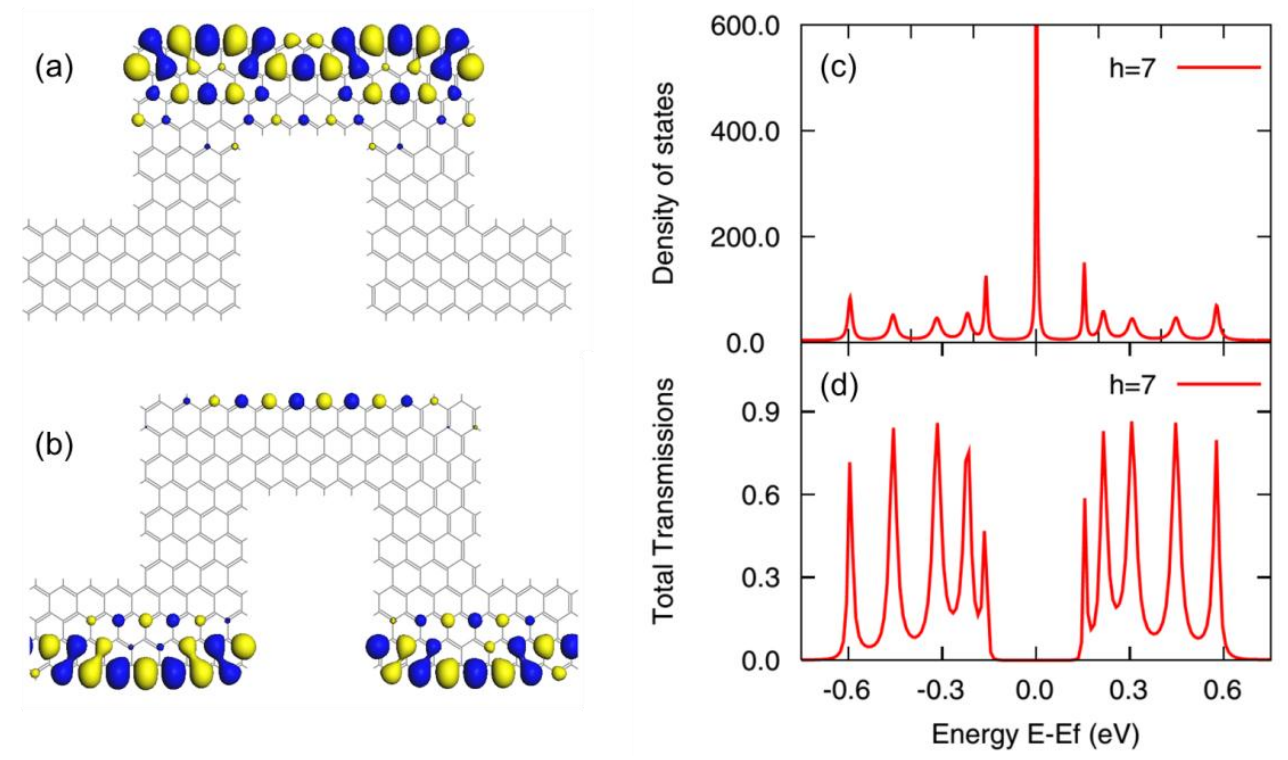

Figure 4. (a) HOMO state, (b) LUMO state, (c) density of state, and (d) transmission spectrum of U-shaped ZGNR junction (with $\mathrm{h}=7$ )

To analyze the transport properties, the DOS and corresponding transmission spectrum of the Ushaped ZGNRs are shown in the Fig. 4(c) and 4(d). It is found that, resonant peaks symmetrically appear at the two sides of Fermi level $(E=0)$ in the transmission spectrum (Fig. 4(d)). Each resonant peak corresponds to a DOS peak except the DOS peak at Fermi level (Fig. 4(c)). The previous firstprinciples calculations demonstrated that the localized states at zigzag edges make a remarkably sharp peak in the DOS at the Fermi level because of intrinsic band structure of Graphene [13]. The lifetime of such the localized state is extremely long [14]. It means that the electrons at the state is almost confined and do not contribute on conductance. Therefore, the DOS peak at Fermi level cannot be seen in transmission spectrum.

\subsection{Gas sensors based on U-shaped GNRs}

As mentioned above, the electronic and transport properties of U-shaped ZGNR indicate that the MOs states around Fermi level are quasi-bound states localized at the zigzag edges. The electrons at these localized states are almost confined and do not contribute on conductance. Besides, the adsorption of $\mathrm{CO}_{2}$ and $\mathrm{NH}_{3}$ on straight AGNRs with double DB defects on edges show that $\mathrm{CO}_{2}$ and $\mathrm{NH}_{3}$ adsorbed molecule contribute additional states in the valance band and conductance band, 
respectively. These additional states are localized and make very sharp peaks in the DOS (Fig. 3(a) and Fig. 3(b)) and also do not contribute on conductance around Fermi level.

In this part, a gas sensor based on U-shaped GNRs was designed, as Fig. 5(a), in which the geometrical structure of the sensor was split to three regions (1), (2), and (3). In particular, region (1) has geometrical structure like semi-infinite Graphene and play as electrodes role. The U-shaped GRN junction includes regions (2) and (3). They have geometrical structures of finite ZGNR or AGNR depending on the edge structures. For U-shaped ZGNR junction, as shown in Fig. 5(a), region (2) is ZGRN and region (3) is AGNR. While, the U-shaped AGNR junction is reverse. For such of the sensor structure, without adsorption, the quasi-bound states (around Fermi level) localized at the zigzag edges. With adsorption of gas molecule such as $\mathrm{CO}_{2}$ or $\mathrm{NH}_{3}$ on armchair edges, which have DB defects, the additional states are appeared and localized at the armchair edges. The two kind of localized states can be "connected" together for making delocalized states on whole of the U-shaped GNR junction. The electrons at these delocalized states are not confined and can be contributed on conductance.

(a)

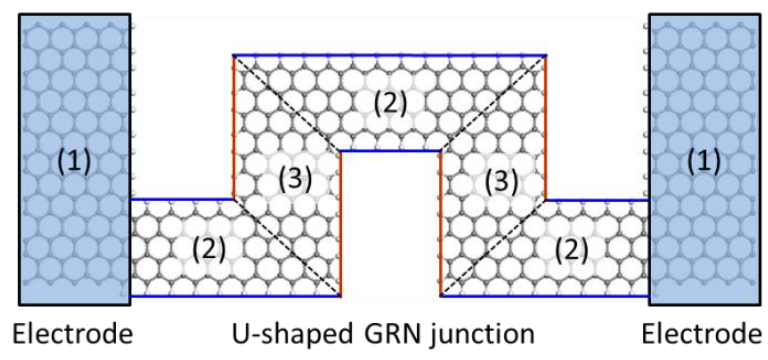

(b)
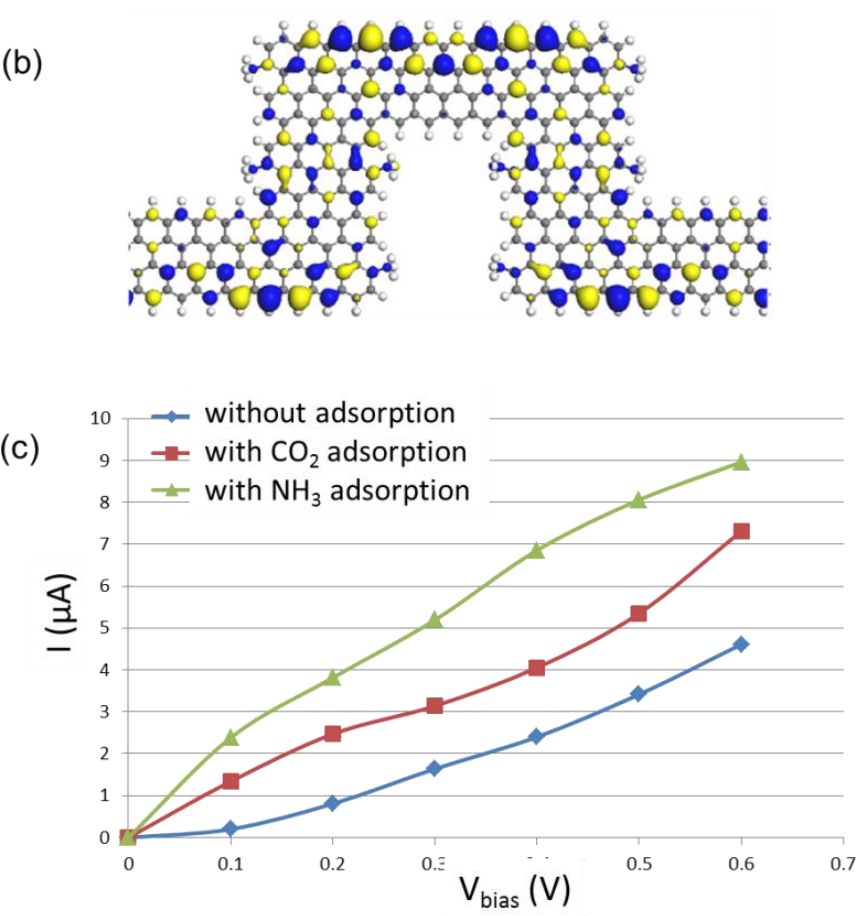

Figure 5. (a) structure model of Gas sensors based on U-shaped GNRs, (b) HOME state of U-shaped ZGRNs, and (c) I-V curve of the U-shaped ZGNR gas sensor. 
Fig. 5(b) shows HOMO state of the U-shaped ZGRN junction with $\mathrm{NH}_{3}$ adsorbed on vertical armchair edges. It is clear see that the localized state at horizontal zigzag edges are connected to the additional states at vertical armchair edges for making a delocalized state. It means that the electrons in the state therefore have more probabilities to escape to the electrodes through the U-shaped junction. In other words, the current is increase with adsorption. Fig. 5(c) shows I-V characteristics of the U-shaped AGNR gas sensor with/without adsorption of $\mathrm{CO}_{2}$ and $\mathrm{NH}_{3}$. We can see that, with adsorption of both $\mathrm{CO}_{2}$ and $\mathrm{NH}_{3}$, currents are significantly increased in comparison with no adsorption. Moreover, the $\mathrm{I}-\mathrm{V}$ curves of $\mathrm{NH}_{3}$ adsorption, $\mathrm{CO}_{2}$ adsorption and without adsorption are totally separated together even with very small bias voltage. In our previous study, a 10-AGNR, which has a band gap around $1.0 \mathrm{eV}$, was used as a junction of a gas sensor [7]. The I-V curves of $\mathrm{NH}_{3}$ adsorption, $\mathrm{CO}_{2}$ adsorption and without adsorption are only separated in region of bias voltage large than $1.4 \mathrm{eV}$. The corresponding currents at the bias voltage of $1.4 \mathrm{eV}$ are very low (smaller than $2 \mu \mathrm{A}$ ). Consequently, the sensitivity and selectivity of the U-shaped GNRs gas sensor is significantly improved in comparison with the pristine GNR ones.

\section{Conclusions}

Based on the calculated results of adsorption of $\mathrm{CO}_{2}$ and $\mathrm{NH}_{3}$ on straight GNRs with DB edge defects and the electronic and transport properties of the U-shaped GNR junctions, gas sensors based on U-shaped GRNs have been designed. The MOs and I-V characteristics were analyzed. It is found that, for U-shaped ZGRNs without adsorption, the states around Fermi level are quasi-bound states localized at the zigzag edges. The electrons at these localized states are almost confined and do not contribute on conductance. However, for U-shaped GRNs with $\mathrm{CO}_{2}$ and $\mathrm{NH}_{3}$ adsorbed on DB armchair edge defects, these states become delocalized. The I-V characteristics show that the sensitivity and selectivity of the U-shaped GNRs gas sensors are significantly improved in comparison with the pristine GNR ones. The obtained results can be used for predicting in experimental fabrication of Graphene based gas sensors.

\section{Acknowledgments}

The author thanks the Vietnam National University (VNU) Hanoi for funding this work within project No. QG.17-12. The computations presented in this study was performed at the Faculty of Physics of VNU University of Science, Vietnam, and the Information Center of Japan Advanced Institute of Science and Technology, Japan.

\section{References}

[1] K.S. Novoselov, A.K. Geim, S.V. Morozov, D. Jiang, Y. Zhang, S. V. Dubonos, I. V. Grigorieva, A. A. Firsov, Electric Field Effect in Atomically Thin Carbon Films, Science 306 (2004) 666-669.

[2] F. Schedin, A.K. Geim, S. V. Morozov, E. W. Hill, P. Blake, M. I. Katsnelson, and K. S. Novoselov, Detection of individual gas molecules adsorbed on graphene, Nature Materials 6 (2007) 652-655

[3] B. Huang, Z. Li, Z. Liu, et al., Adsorption of gas molecules on graphene nanoribbons and its implication for nanoscale molecule sensor, The Journal of Physical Chemistry C 112 (35) (2008) 13442-13446. https://doi.org/10.1021/jp8021024

[4] O. Leenaerts, B. Partoens, F.M. Peeters, Adsorption of $\mathrm{H}_{2} \mathrm{O}, \mathrm{NH}_{3}, \mathrm{CO}, \mathrm{NO}_{2}$, and $\mathrm{NO}$ on graphene: A firstprinciples study, Phys. Rev. B. 77 (2008) 125416. https://doi.org/10.1103/PhysRevB.77.125416

[5] H.E. Romero, P, Joshi, A.K. Gupta, H.R. Gutierrez, M.W. Cole, S.A. Tadigadapa, P.C. Eklund, Adsorption of ammonia on graphene, Nanotechnology 20 (2009) 245501-245509. Doi: 10.1088/0957-4484/20/24/245501 
[6] A.K. Mishra, S. Ramaprabhu, Carbon dioxide adsorption in graphene sheets, AIP Advances 1, (2011) 032152. https://doi.org/10.1063/1.3638178

[7] Nguyen Tien Cuong, Nguyen Manh Tien, First-principles Studies of $\mathrm{CO}_{2}$ and $\mathrm{NH}_{3}$ Gas Molecules Adsorbed on Graphene Nanoribbons, VNU Journal of Science: Mathematics - Physics 32(2) (2016) 15-21.

[8] J.A. Robinson, E.S. Snow, S.C. Badescu, T.L. Reinecke, Role of defects in single-walled carbon nanotube chemical sensors, Nano Lett., 6(8) (2006) 1747-1751. https://doi.org/10.1021/n10612289

[9] J. Andzelm, N. Govind, A. Maiti, Nanotube-based gas sensors - Role of structural defects", Chemical Physics Letters, 421(1-3) (2006) 58-62. https://doi.org/10.1016/j.cplett.2005.12.099

[10] Nguyen Tien Cuong, Hiroshi Mizuta, Bach Thanh Cong, Nobuo Otsuka, and Dam Hieu Chi, "Ab-initio Calculations of Electronic Properties and Quantum Transport in U-shaped Graphene Nanoribbons" International Journal of Computational Materials Science and Engineering 1(3) (2012) 1250030. https://doi.org/10.1142/S2047684112500303

[11] Available online at https://www.3dsbiovia.com/products/collaborative-science/biovia-materials-studio/

[12] Available online at http://www.openmx-square.org/

[13] K. Nakada, M. Fujita, Edge state in graphene ribbons: Nanometer size effect and edge shape dependence, Phys. Rev. B 54 (1996) 17955. https://doi.org/10.1103/PhysRevB.54.17954

[14] Y.P. Chen, Y.E. Xie, J. Zhong, Resonant transport and quantum bound states in Z-shaped graphene nanoribbons, Phys. Lett. A 372 (2008) 5928-5931. https://doi.org/10.1016/j.physleta.2008.07.058 